\title{
Multilinguales
}

Pratiques littéraires, linguistiques, pédagogiques, didactiques et médiations culturelles contemporaines

\section{La compétence bi/plurilingue en classe de langue en Algérie}

The Bi/Multilingual Competence in Language Classes in Algeria

\section{Nabila Tatah}

\section{OpenEdition}

\section{Journals}

Édition électronique

URL : https://journals.openedition.org/multilinguales/3255

DOI : 10.4000/multilinguales.3255

ISSN : 2335-1853

Éditeur

Université Abderrahmane Mira - Bejaia

Édition imprimée

Date de publication : 1 juin 2013

Pagination : 121-131

ISSN : 2335-1535

\section{Référence électronique}

Nabila Tatah, « La compétence bi/plurilingue en classe de langue en Algérie », Multilinguales [En ligne], 1 | 2013, mis en ligne le 01 juin 2013, consulté le 01 juillet 2021. URL : http://journals.openedition.org/ multilinguales/3255; DOI : https://doi.org/10.4000/multilinguales.3255

Ce document a été généré automatiquement le 1 juillet 2021.

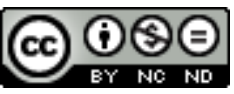

Multilinguales est mise à disposition selon les termes de la Licence Creative Commons Attribution Pas d'Utilisation Commerciale - Pas de Modification 4.0 International 


\title{
La compétence bi/plurilingue en classe de langue en Algérie
}

\author{
The Bi/Multilingual Competence in Language Classes in Algeria
}

\author{
Nabila Tatah
}

1 La conception de l'enseignement de la langue étrangère à travers les différentes méthodologies qui se sont succédé, depuis les années cinquante, a beaucoup contribué à l'évolution de la didactique des langues. Dans la méthodologie traditionnelle, appelée aussi grammaire traduction, on avait recours à la technique de la traduction par le procédé de thème/version, tandis que le recours à la langue première était interdit dans les autres méthodologies. La méthodologie directe constituait une approche naturelle de l'apprentissage d'une langue étrangère fondée sur l'observation de l'acquisition, par l'enfant, de la langue maternelle. Par cette méthode, on recourait à l'ensemble des procédés et techniques permettant d'éviter le recours à la langue maternelle; ce qui a entraîné un bouleversement dans l'enseignement des langues étrangères. Ainsi, les méthodologies retenues successivement pour l'apprentissage des langues étrangères ne réservent pas le même sort à la langue maternelle.

D'abord, dans la méthodologie audio-orale (MAO), les habitudes linguistiques de la langue maternelle sont principalement considérées comme une source d'interférences lors de l'apprentissage, en classe, d'une langue étrangère. Pour les contourner, il était recommandé de n'utiliser que la langue étrangère.

3 Dans la méthodologie structuro-globale audio-visuelle (SGAV), ensuite, le contexte social d'utilisation d'une langue était pris en considération. Celle-ci aurait, certes, permis d'apprendre assez vite à communiquer oralement avec des natifs de langues étrangères. Mais elle n'offrait pas la possibilité de comprendre ni des natifs parlant entre eux, ni les médias.

Quant aux approches communicatives, elles autorisent l'enseignant, théoriquement du moins, à faire référence à la langue maternelle, lorsque le besoin s'en fait sentir en classe. En cours de langue, on utilise de préférence la langue étrangère, mais il est possible de faire appel à la langue maternelle (C. Puren, $1988: 50$ ). 
5 Ce parcours historique nous révèle certes, que les objectifs de l'apprentissage d'une langue étrangère et la place réservée à la langue maternelle ont beaucoup varié dans le temps, mais aussi que la recherche dans le domaine de la didactique des langues est en constante évolution. Actuellement, on considère que le milieu scolaire constitue un champ privilégié de contact entre les langues et les cultures dans une société donnée qui, en général, est bi/plurilingue et pluriculturelle.

\section{Bilinguisme et enseignement des langues}

6 Il faut reconnaître qu'aujourd'hui avoir recours à une autre langue que sa langue

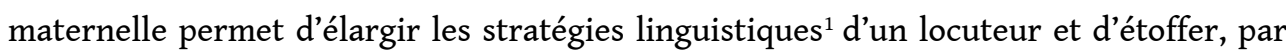
là même, son discours métalinguistique ${ }^{2}$. M. Causa explique aussi, à ce sujet, que la réalité dans le domaine de l'enseignement montre que l'alternance codique employée par l'enseignant est une pratique naturelle conforme à toute situation de communication de contact de langues.

7 Cette pratique langagière ne va pas non plus à l'encontre des processus d'apprentissage : elle constitue au contraire un procédé de facilitation parmi d'autres, selon le même auteur: " pour faciliter l'accès à la langue cible, l'enseignant emploie l'autre code qui "circule" dans la classe ». (M. Causa, citée par S. Ehrhart, 2002 : 3)

8 Cette orientation nouvelle de la méthode d'apprentissage des langues a été décelée quelques années plus tôt par Louise Dabène (1994) : « On tend de plus en plus à considérer le parler du bilingue comme un ensemble original dont il eut été plus bénéfique d'étudier le fonctionnement que de repérer les déviances ou les insuffisances par rapport aux normes standard des langues concernées » (87).

9 Dans cette optique, l'alternance codique, " c'est-à-dire les passages dynamiques d'une langue à l'autre, est l'une des manifestations les plus significatives du parler bilingue » (Causa, 2007 :18), doit donc être considérée comme stratégie d'enseignement/apprentissage dans des situations de forte demande d'échange communicatif (S. Ehrhart, $2002: 4$ ).

10 Chez les enseignants, la stratégie du code-switching, qui désigne la capacité volontaire à faire intervenir une deuxième langue dans le discours, a des fins didactiques: ils alternent les langues afin de mieux expliquer une consigne ou une leçon (plan du contenu), de structurer le déroulement d'un cours (plan didactique), ou de se rapprocher des apprenants (plan émotionnel, relationnel) (S. Ehrhart, 2002).

\section{L'approche plurilingue : problématique et méthodologie}

11 En Algérie, jusqu'en 2010, l'utilisation de la L1 était tolérée dans les classes de langues. Aujourd'hui, cette pratique est officialisée (Article 4 de la loi d'orientation de 2010, $\mathrm{n}^{\circ}$ 77/0.0.3/10): l'utilisation de la traduction dans l'enseignement des langues étrangères est autorisée; ce qui constitue, à notre avis, un moyen de faciliter la compréhension du cours par les apprenants.

12 Mais, très souvent aussi, face au plurilinguisme et au pluriculturalisme de la classe, les enseignants sont assez dépourvus parce que cette situation requiert des compétences linguistiques et culturelles spécifiques. Dès lors, ces compétences se construisent dans 
un contexte extrascolaire et il s'avère difficile de les réinvestir dans les pratiques scolaires.

13 Ainsi, notre démarche, qui ne peut être qu'expérimentale, repose sur le principe que les apprenants doivent avoir la possibilité de réinvestir, en toute circonstance, leurs acquis langagiers antérieurs issus de leur environnement plurilingue et pluriculturel ou de leur apprentissage scolaire. Nous avons choisi, en cela, de nous inscrire dans l'approche plurilingue telle que définie par le Conseil de l'Europe :

L'approche plurilingue met l'accent sur le fait que, au fur et à mesure que l'expérience langagière d'un individu dans son contexte culturel s'étend de la langue familiale à celle du groupe social puis à celle d'autres groupes (que ce soit par apprentissage scolaire ou sur le tas), il/elle ne classe pas ces langues et ces cultures dans des compartiments séparés mais construit plutôt une compétence communicative à laquelle contribuent toute connaissance et toute expérience des langues et dans laquelle les langues sont en corrélation et interagissent. (Conseil de l'Europe, 2001 : 11)

14 Conformément aux principes de cette approche, et du fait de leurs acquis langagiers antérieurs à leur scolarisation, les enfants algériens scolarisés ont, non seulement la possibilité de réinvestir leurs acquis scolaires et langagiers, mais aussi, leur répertoire plurilingue. Selon Hamers et Blanc (1983) : «l'alternance de codes (code-switching) est une stratégie de communication utilisée par des locuteurs bilingues entre eux; cette stratégie consiste à faire alterner des unités de longueur variable de deux ou plusieurs codes à l'intérieur d'une même interaction verbale » (199).

15 Cependant, il y a lieu de noter que cette stratégie communicative, dont traitent Hamers et Blanc, constitue une source complexe pour les apprenants bilingues qui sont appelés à mettre en œuvre des stratégies verbales (changement de code, emprunt, paraphrase, etc.), pour donner un sens à leur discours. A cet effet, les sociolinguistes s'accordent à présenter la situation linguistique algérienne comme étant une situation plurilingue, dans laquelle différentes langues interagissent, comme l'arabe (aussi bien dans sa forme dite classique que dans sa forme dialectale), le berbère (avec toutes ses variantes), ainsi que le français (Morsly, 1996, Taleb-Ibrahimi, 1995).

16 Nous avons choisi d'observer deux classes mixtes de 1 èreannée secondaire, composées d'une vingtaine de filles et d'une vingtaine de garçons entre quinze et dix-sept ans, dans un lycée du vieux quartier de la ville de Bejaia (dit « la haute ville»), sur la côte est de l'Algérie. Le public concerné, par cette étude ${ }^{3}$, qui aborde la huitième année d'apprentissage du français, est issu de milieux sociaux différents et son niveau de compétence en langue française est hétérogène. En outre, ce que nous savions, au préalable, de ces adolescents, pour l'avoir bien observé dans le hall du lycée et même en dehors de l'enceinte de l'établissement, c'est qu'ils utilisent systématiquement et alternativement, dans leurs communications quotidiennes, un parler bilingue : arabe/ français, kabyle/français, arabe/kabyle.

17 Les séances d'observation nous ont permis de recueillir un corpus oral constitué de discours et de conversations entre l'enseignant et les apprenants, entre les apprenants. Pendant nos séances d'observation, nous avons choisi de ne retenir que les interventions au cours desquelles l'usage des différentes langues est pertinent pour l'objet de notre recherche. Dans la transcription de notre corpus, nous désignons par 'E1', le premier enseignant, et par 'E2', le second. Nous utilisons, par ailleurs, l'abréviation 'A' pour l'apprenant, et 'AA', les groupes d'apprenants. 
Pour ce qui est de la transcription, en elle-même, nous nous sommes inspirée des conventions de Valibel et Gars (Dister et al. 2008), complétées par des rajouts imposés par les spécificités linguistiques du public de notre étude. Les passages transcrits sont à chaque fois repris dans les trois langues d'usage : arabe, kabyle et français.

\section{L'expérimentation de l'approche plurilingue}

19 L'enseignant, en contexte scolaire, exerce un contrôle important sur l'interaction : il sélectionne la tâche, régule les thèmes, travaille activement à la facilitation du déroulement des échanges avec des apprenants en situation d'apprentissage, et doit veiller au maintien de l'intercompréhension tout en visant le progrès de l'apprenant dans la langue cible. Pour l'analyse de son recours à la L1, nous nous référons aux travaux de Sabine Ehrhart qui énumère trois grands types de comportements dans le choix des langues chez les enseignants :

Le puriste qui refuse tout emploi de la L1 par les élèves, Le modéré qui tolère la L1 avec ou sans intégration de celle-ci dans la conversation, L'utilisateur actif dont l'emploi actif peut être provoqué par l'utilisation de la L1 par les élèves ; mais ce cas de figure ne se présente pas souvent (Ehrhart, 2002 :6).

\section{La compétence bilingue/plurilingue}

20 Pour communiquer, les sujets bilingues ou plurilingues disposent de plusieurs langues. Ces langues ne sont pas utilisées indifféremment, mais en fonction de la situation de communication. D. Moore (2006) considère que les locuteurs qui vivent avec plusieurs langues « utilisent les langues [...] à leur disposition, pour des besoins de communication précis et différenciés dans des contextes sociaux spécifiques" (98). On parle alors de compétences bilingue ou plurilingue.

21 Les personnes bi-/plurilingues sont :

- les personnes qui se servent de deux ou plusieurs langues (ou dialectes) dans la vie de tous les jours. Sont comprises les personnes qui ont une compétence de l'oral dans une langue, et une de l'écrit dans une autre,

- les personnes qui parlent plusieurs langues avec un niveau de compétence différent dans chacune d'elles (qui ne savent ni lire ni écrire dans l'une ou l'autre),

- les personnes qui possèdent une maîtrise parfaite de deux ou plusieurs langues (F.Grosjean, 1993)

La personne bi-/plurilingue se caractérise par trois traits distinctifs :

- elle participe, au moins en partie, à la vie de deux cultures (deux mondes, des réseaux culturels majeurs, deux environnements culturels) et ceci de manière régulière

- elle sait adapter, partiellement ou de façon plus étendue, son comportement, ses attitudes, son langage à un environnement culturel donné

- elle combine et synthétise les traits de chacune des deux cultures.

23 En effet, certains traits (attitudes, croyances, valeurs, goûts et comportements) proviennent de l'une ou l'autre culture et se combinent, tandis que d'autres n'appartiennent plus ni à l'une ni à l'autre, mais sont la synthèse des deux.

C'est cet aspect de synthèse qui reflète sans doute le mieux l'être biculturel. Castellotti,

Coste et Moore pensent que : 
la désignation compétence plurilingue porte sur la compétence à communiquer d'acteurs sociaux en mesure d'opérer, à des degrés variables, dans des langues différentes. Il s'agit d'une compétence dynamique, dans le sens où ces acteurs sont à même aussi de gérer et remodeler cette compétence plurilingue au cours de leur trajectoire personnelle, en fonction de leurs besoins et des situations. (2001:102) bilingue », " alternance codique » ou "code-switching», se caractérise par un usage alterné de deux ou plusieurs langues, par un ou plusieurs locuteurs, à l'intérieur d'un échange de parole. Cette alternance codique peut revêtir différentes formes :

- l' « alternance intralocuteur » : passer d'une langue à l'autre,

- l' "alternance interlocuteur»: un individu parle dans une langue et un autre lui répond dans une autre langue,

- l' «alternance intraphrase » : appelée aussi « mélange codique » ou « code-mixing»: deux langues sont présentes à l'intérieur d'une même phrase,

- l' «alternance traductive »: une phrase est produite dans une langue puis traduite dans une autre,

- l' «alternance continue»: passage d'une langue à l'autre sans couper le fil de la conversation.

Les exemples qui illustrent cette étude relèvent de ces types d'alternance.

\section{Les interactions enseignant / apprenants}

Dans les interactions enseignant/apprenant, nous avons constaté que le recours des enseignants à la L1 est souvent lié à leurs compétences linguistiques, sans pour autant y être entraînés par leurs élèves. En fait, ils ne l'utilisent pas seulement pour se rapprocher d'eux, mais aussi pour détendre l'atmosphère de la classe. C'est ainsi que nous avons interprété les différentes expressions qu'ils utilisent pour saluer les élèves en entrant en classe, le matin: Azoul (tamazight), s'bah:elkheir (arabe dialectal), essa'laamou :aleykoum (arabe classique), bonjour (français).

L'enseignant peut, parfois, avoir recours à la L1 de façon directe ou indirecte :

10. A6 - c'est-à-dire la famine / zaa'ma elmaja'a : l'enseignante acquiesce de la tête pour approuver l'énoncé équivalent en arabe.

L'enseignant a recours aussi à ce procédé pour lever l'ambiguïté et assurer une meilleure intercompréhension au sein de la classe. Par exemple, lorsqu'un élève demande le sens du mot 'rudimentaire', l'enseignante donne plusieurs synonymes en langue cible et pour s'assurer d'une meilleure transmission du message, elle finit par avoir recours à la traduction en langue arabe :

14. E1- en arabe standard on dit $\mathrm{kadi} / \mathrm{m} \uparrow$

13. A8- madame // que veut dire rudimentaire? (...) 
14. E1: - ça veut dire que les méthodes employées sont très simples, anciennes, primitives (...) en arabe standard on dit $\mathrm{kadi} / \mathrm{m} \uparrow$

31 A ce stade de notre étude, on n'ignore donc pas que dans leurs pratiques de classe, les enseignants font appel à plusieurs stratégies suivant les besoins réels de la communication. Mais il n'est pas facile pour nous de parler de choix effectif de langue car, comme nous avons pu l'observer, cet acte n'est pas toujours conscient. Nous avons bien interrogé les enseignants sur ce qui motivait ce passage de la langue cible à la langue première et, à travers leurs réponses, nous nous sommes rendu compte que bien souvent ils se trouvaient dans une zone d'ombre, entre les deux langues ${ }^{4}:$ L1 et L2 :

E1 : - Je ne me rends pas toujours compte de cette situation de transfert de la L2 vers la L1 // les élèves sont parfois épuisants // Euh / peut-être aussi que le message passe mieux lorsque je m'exprime dans ma langue maternelle(...).

E2 : - les effectifs chargés des classes font que l'on perde le contrôle parfois // Euh. / Cela m'échappe // cela m'arrive aussi quand je suis excédée par certains comportements de mes élèves.

Rappelons que nous avons affaire ici, à un public d'adolescents, par conséquent l'enseignant doit non seulement jouer son rôle de pédagogue mais doit aussi agir en tant qu'éducateur (rappel des règles de vie et de conduite les plus simples qu'il n'hésite pas à formuler dans la L1).

Nous avons également noté des fluctuations dans le comportement d'un enseignant, dans des situations quasi identiques.

Mais, très souvent, c'est le passage d'une situation plus formelle à une situation plus naturelle dans l'emploi de deux langues qui déclenche le changement de langue. Aussi, nous avons observé une plus haute fréquence dans le recours de l'enseignant au codeswitching ou à l'alternance codique, à la fin des cours, comme dans une situation non protocolaire, hors du cadre institutionnel. Dans ce cas, l'alternance codique employée par l'enseignant constitue une stratégie de communication qui ne va pas à l'encontre du processus d'apprentissage. C'est ce que suggèrent les exemples suivants :

- E1-Soukout! (arabe classique), essa/ktou! (arabe dialectal), souss/mem (kabyle), taisezvous! (français). Il s'agit ici, d'un rappel à l'ordre de l'enseignant pour calmer les élèves agités à la fin d'un cours.

- E1-jii/b l'kta/ab (arabe dialectal), awed// thaktoubth (tamazight), donne-moi le livre! (français). Ce sont les propos de l'enseignant qui passe dans les rangs, à la fin de la séance, pour récupérer les livres empruntés à d'autres élèves.

Selon D. Moore, l'enseignant peut s'appuyer sur la langue de l'apprenant pour le motif du maintien de la communication, ou pour aider celui-ci à franchir les ponts qui vont l'amener vers l'apprentissage de la langue-cible. La L1 joue, ainsi, plusieurs rôles :

- elle fonctionne comme une balise du dysfonctionnement (dans le sens où, le recours à L1 signale un manque à combler dans la langue cible) ;

- elle permet la focalisation sur le lieu de détresse et l'appel à l'aide qui pousse l'enseignant à construire une stratégie de réparation ;

- elle permet de maintenir le contact (la communication);

- elle représente un passage ouvert vers l'autre langue (en établissant des ponts entre elles). (Moore, 1996 :161) 


\section{Les interactions entre apprenants}

de classe, le changement de langue représente un moyen efficace pour focaliser l'attention du maitre sur un besoin ponctuel dans l'effort de production. L'apprenant, lorsqu' il se trouve dans la difficulté de comprendre le message, peut opter pour le recours à la L1 pour baliser le secteur qui lui pose problème dans le seul but de maintenir la communication, comme on peut le voir dans cet exemple :

9. A5- dachou zaa'ma la famine $\downarrow$ (que veut dire le mot famine ?) [rétorque son camarade en kabyle]

Pour Jisa et Richaud (1994), ces séquences de clarification et les reformulations dans la conversation « constituent des cas permettant d'examiner la manipulation de la langue dans le comportement intentionnel de l'enfant: ce dernier est focalisé sur un but communicatif ; il emploie des moyens alternatifs pour l'atteindre lorsqu'il estime que le moyen initial ne réussit pas » (29).

L'apprenant peut avoir recours directement au changement de langue, sans préparation préalable, ou au contraire amener progressivement la possibilité d'une ouverture bilingue, en cherchant l'assentiment de l'enseignant :

11. A7 [à cause $\mathrm{nl} /$ misirya à m'dame $\uparrow$ ] (à cause de la misère, Madame.) [Chuchotements inaudibles au fond de la salle]

L'apprenant, en faisant référence à la misère, tente d'expliquer, ici, les causes de la sous-alimentation en ayant recours à une interférence.

Nous avons relevé également, lors de nos séances d'observation, que les élèves qui disposent d'un bon niveau en langue aident les moins bons à comprendre et à s'exprimer dans la langue cible.

41 Ils interviennent, le plus souvent, en usant de l'interlangue pour corriger ou expliquer des éléments du cours à leurs camarades en difficulté :

8. A4 - non $\uparrow$ pas tous les gens / il y a la famine dans le monde $\downarrow$

9. A5- dachou zaa'ma la famine $\downarrow$ (que veut dire « famine »?)

10. A6 -zaa/ma elmaja/a [traduction du mot «famine» en arabe par un autre camarade]

Les sujets bilingues ou plurilingues ont donc comme point commun, une capacité à communiquer efficacement en fonction de l'interlocuteur et de la situation de communication. L'une des caractéristiques des compétences des sujets bilingues ou plurilingues est la capacité à passer d'une langue à l'autre et à activer les deux (ou plus de deux) systèmes linguistiques. On observe alors dans les échanges «des mélanges de langues» (Cadiot, $1987: 50)$.

\section{Le mélange des langues}

43 Comme nous l'avons vu, les sujets bilingues ou plurilingues ont à leur disposition plusieurs codes linguistiques pour s'exprimer; ils utilisent les langues de leur répertoire en fonction de leur interlocuteur et de la situation de communication.

Dans leurs pratiques langagières, on note la présence de la langue 1 dans la langue 2 qui se manifeste par ce que Lüdi et Py (2003) appellent : «des marques transcodiques » qu'ils 
définissent comme "tout observable, à la surface d'un discours en une langue ou variété donnée, qui représente, pour les interlocuteurs et/ou le linguiste, la trace de l'influence d'une autre langue ou variété" (142). Ces «marques transcodiques» peuvent prendre différentes formes : interférences, emprunts, code-switching, code-mixing (Weinreich, $1953: 59-60), \ldots$

45 En situation monolingue, le locuteur bilingue choisit la langue de l'interlocuteur sauf s'il souhaite établir volontairement une distance par rapport à lui. Comme l'indiquent Lüdi et Py:» le choix du mode bilingue correspond à une option de définition de la situation [...], les énoncés sont émaillés de nombreux mélanges de langues, [...] manifestant l'activation plus ou moins simultanée des deux systèmes linguistiques » (Lüdi et Py, 2003 : 140) :

4. A1 : tu as compris? [L'apprenant s'adressant à son camarade de table] neki oufhim : ghara/dachou an / aal dayé $\downarrow$ (en kabyle) (je n'ai pas compris ce qu'il faut faire ici.) explique-moi si tu as compris (...)

5. A2 : on doit décrire ce qu'il y a sur l'image, akhdhem/ akhdhem/ (...). [L'apprenant ordonne à son camarade de travailler].

46 Ainsi, les mélanges de langues sont une caractéristique du locuteur bilingue. Ils font, cependant encore souvent, l'objet de représentations négatives. Gadet et Varro soulignent que, les bilingues s'avèrent aussi pratiquer ce qui a longtemps été tenu pour la «part noire» du bilinguisme: les parlers mixtes. Tous les termes désignant ce phénomène, pourtant très courant, ont des connotations dépréciatives (mélange, métissage, hybridation, contamination) et ont le défaut de traiter les deux langues comme des entités stabilisées (Gadet et Varro, 2006 :15).

Les quelques cas de la compétence bilingue/plurilingue, présentés ici, nous ont permis d'illustrer quelques-unes des stratégies employées par les enseignants et les apprenants pour compenser une carence linguistique en situation de communication. Dans le contexte de l'enseignement/apprentissage des langues, la diversité des situations et des contextes nous incite donc à reconsidérer les moyens qui permettent de développer des atouts d'apprentissage chez les apprenants qui évoluent dans un milieu plurilingue.

\section{BIBLIOGRAPHIE}

ANCIAUX, F, « Vers une didactique de l'alternance codique en éducation aux Antilles françaises », in RIVIÈRE, V, (Ed.), Spécificités et diversité des interactions didactique, Paris, Editions Riveneuves, pp. 97-112, 2012.

CASTELLOTTI, V, COSTE, D. \& MOORE, D, « Le proche et le lointain dans les représentations des langues et leur apprentissage », in, MOORE, D (éd.) Les Représentations des langues et leur apprentissage : référence, modèles, données et méthodes), Paris, Didier, pp. 101-131, 2001.

CAUSA, M, «L'alternance codique dans le discours de l'enseignant », " Les Carnets du Cediscor », pp. 111-129, 1996. Disponible sur le site : [http://cediscor.revues.org/404].

CAUSA, M, L'alternance codique dans l'enseignement d'une langue étrangère - Stratégies

d'enseignement bilingues et transmission de savoirs en langue étrangère, Peter Lang, 2002. 
CADIOT, P, « Les mélanges de langue », in Vermes, G., Boutet, J. (éds.), France, pays multilingue. t. 2 : Pratiques des langues en France, Paris, l'Harmattan, pp. 50-61, 1987.

Cadre européen commun de référence pour les langues. Apprendre, Enseigner, Evaluer, Conseil de la Coopération Culturelle, Comité de l'Education, Division des Langues Vivantes, Strasbourg, 2001.

CUQ, J, P, Dictionnaire de didactique du français langue étrangère et seconde, asdifle, CLE International, 2003.

DABENE, L, Repères sociolinguistiques pour l'enseignement des langues, Paris, Hachette, 1994.

DISTER, et al, Conventions de transcription régissant les corpus de la banque de données VALIBEL. Disponible sur le site : [http://valibel.fltr.ucl.ac.be

EHRHART, S, «L'alternance codique dans le cours de langue : le rôle de l'enseignant dans l'interaction avec l'élève - Synthèse à partir d'énoncés recueillis dans les écoles primaires de la Sarre ». Disponible sur le site : www.ph-karlsruhe.de/fileadmin/user.../EHRHART-code-swt-LV.pdf GADET, F, et VARRO, G, « Le scandale du bilinguisme », dans Langage \& société, n 116, pp. 10-24, 2006.

GROSJEAN, F, Le bilinguisme et le biculturalisme : essai de définition, Tranel, 19, pp. 13-42, 1993.

HAMERS, J, F, et BLANC, M, Bilingualité et bilinguisme, Mardaga, 1983.

HYMES, D, Vers la compétence de communication, Paris, Didier, 1991.

JISA, H. \& RICHAUD, F, Quelques sources de variations chez les enfants. Acquisition et Interaction en Langue Etrangère, (4), p367-376,1994. Disponible sur le site : [http://aile.revues.org/1251].

LÜDI, G, \& PY, B, Être bilingue, (3e éd). Berne, Peter Lang, 2003.

Ministère de l'Education Nationale, Loi d'orientation de 2010, n 77/0.0.3/10 », Alger, 2010.

MOORE, D, Plurilinguismes et école, Collection LAL, Paris, Editions Didier, 2006.

MORSLY, D, « Alger plurilingue », dans Plurilinguismes, n 12, 1996, pp. 47-80.

PERRAULT, M, Analyse du discours métalinguistique des enseignants de français comme révélateur de leur conceptualisation des notions linguistiques enseignées. Mémoire en linguistique, Université de Montréal, Août 2006. Disponible sur le site : [http://olst.ling.umontreal.ca/pdf/

PerraultMA2006.pdf]

PUREN, C, Histoire des méthodologies de l'enseignement des langues, Paris Nathan- Clé International, 1988.

TALEB-IBRAHIMI, Kh, Les Algériens et leur(s) langue(s). Éléments pour une approche sociolinguistique de la société algérienne, Alger, Dar El-Hikma, 1995.

WEINREICH, U, Language in contact, La Haye, Mouton, 1953.

\section{NOTES}

1. Une stratégie linguistique est ce qui permet de pallier certaines des difficultés que l'on pourrait éprouver dans le maniement de la langue cible (J. P. Cuq, 2003 :225).

2. Le discours métalinguistique est une manifestation, dans l'acte d'énonciation, de l'attitude du locuteur, face à la langue et à ses pratiques (M. Perrault, 2006 :17).

3. Nous tenons à rappeler ici, qu'il s'agit d'une étude que nous avions réalisée dans le cadre du projet d'un recherche (2012), sous la direction du professeur, AREZKI Abdenour. 
4. Dans notre contexte, le parler utilisé par les locuteurs de la L1 peut être soit le kabyle, soit l'arabe dialectal. Quant à la L2, on la définit ici, comme langue étrangère, en l'occurrence, le français, l'anglais, ou encore une autre langue étrangère pour certains apprenants qui ont fait le choix d'une autre langue étrangère.

\section{RÉSUMÉS}

En Algérie, plusieurs langues sont en interaction. Celles-ci se produisent dans tous les milieux, mais nous ne nous intéressons, dans le cadre de notre étude, qu'à celui de l'enseignement. Nous avons tenté de mieux comprendre les modalités de gestion des productions langagières des différents locuteurs en contexte scolaire. Notre analyse est organisée en deux temps : le premier est consacré aux stratégies utilisées par les enseignants et les apprenants, en classe de première année secondaire, et le second aux compétences de communication de ces locuteurs plurilingues et pluriculturels.

In Algeria, many languages are constantly interacting. They may occur in all environments, but we are interested in our study in teaching. We have tried to better understand methods of managing the linguistic productions of different speakers in a school context. Our analysis is organized into two phases: the first is devoted to strategies used by teachers and learners in a first year secondary class. The second one puts focus on communicative competences of these multilingual and multicultural speakers.

\section{INDEX}

Keywords : multilingualism, bilingualism, teaching strategy, code switching, multilingual competence

Mots-clés : plurilinguisme, bilinguisme, stratégie d'enseignement, interaction, alternance codique

\section{AUTEUR}

\section{NABILA TATAH}

Université A. Mira - Bejaia 